\title{
Measurement of the weak nucleon-nucleon interaction by polar- ized cold neutron capture on protons
}

\author{
R. Alarcon ${ }^{1, a}$, D. Blyth ${ }^{1, b}$, and the NPDGamma Collaboration ${ }^{\mathrm{c}}$ \\ ${ }^{1}$ Department of Physics, Arizona State University, Tempe, AZ 85287-1504, USA
}

\begin{abstract}
The NPDGamma Experiment at the Spallation Neutron Source at Oak Ridge National Laboratory is measuring the parity-odd correlation between the neutron spin and the direction of the emitted photon in the capture of polarized cold neutrons on protons. A parity violating asymmetry from this process is directly related to the strength of the hadronic weak interaction between nucleons. The experiment was run first with heavier nuclear targets to check systematic effects, false asymmetries, and backgrounds. Since early 2012 the experiment has been collecting data with a 16-liter liquid parahydrogen target. Data taking will continue through 2013 until statistics for a $10^{-8}$ asymmetry measurement are expected. The experiment performance will be discussed as well as the status of the asymmetry measurements.
\end{abstract}

\section{Motivation}

The experimental study of weak interactions between nucleons has progressed slowly because signals from strong interactions heavily mask the order $10^{-7}$ Parity-Violating (PV) effects. The high precision required is now possible with new high intensity neutron sources. An accurate measurement can help to discriminate among models for PV effects in hadronic systems, and in the process help to shed light on the nature of the short-range strong interaction between nucleons [1].

The immense difficulty of calculating the effects of weak interactions in strongly interacting systems necessitates the development of effective theories utilizing degrees of freedom of hadrons as a whole, rather than the fundamental degrees of freedom of their constituents. The traditional Desplanques, Donoghue, and Holstein (DDH) meson exchange model [2] parametrizes the hadronic weak interaction using six PV couplings,

$$
f_{\pi}^{1}, h_{\rho}^{0}, h_{\rho}^{1}, h_{\rho}^{2}, h_{\omega}^{0}, h_{\omega}^{1}
$$

where the superscript indicates the isospin change, and the subscript refers to the exchange meson.

\footnotetext{
ae-mail: ralarcon@asu.edu

be-mail: dblyth@asu.edu

${ }^{c}$ Arizona State University, Bhabha Atomic Research Centre, Hamilton College, Indiana University, Joint Institute of Nuclear Research, High Energy Accelerator Research Org. (KEK), University of Kentucky, Los Alamos National Laboratory, University of Manitoba, University of Michigan, University of Nevada Las Vegas, University of New Hampshire, Oak Ridge National Laboratory, Paul Scherrer Institute, Shanghai Institute of Applied Physics, University of Tennessee, University of Tennessee Chattanooga, Universidad Autónoma de México, TRIUMF, University of Virginia, Western Kentucky University
} 


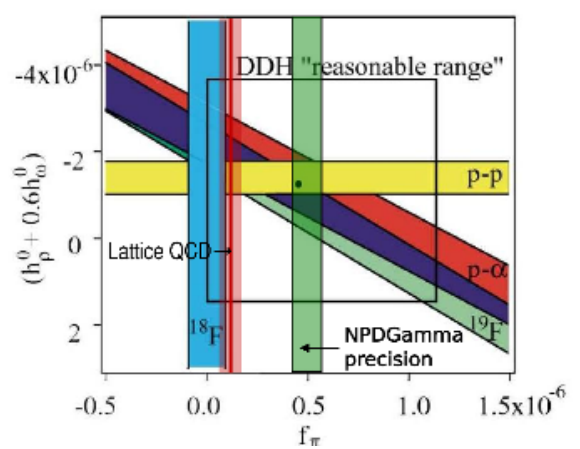

Figure 1. This 2-D exclusion plot of DDH vector vs. scalar couplings shows several previous experimental results, an LQCD calculation [3], and the target NPDGamma precision.

The 2-D exclusion plot in Figure 1 shows some previous experimental results, the DDH prediction, an LQCD calculation [3], and the target NPDGamma precision (not a result). DDH predict a reasonable range (black box) and "best value" (black dot). Previous $f_{\pi}$ measurements come from few-body processes, but the $n p \rightarrow d \gamma$ reaction has the advantage of being a two-body process, providing an unambiguous determination of the pion coupling constant. In addition, the precision of the NPDGamma experiment will help to put the DDH model to a conclusive test with a potential $1 \cdot 10^{-7}$ uncertainty in $f_{\pi}$.

\section{The $n p \rightarrow d \gamma$ PV Asymmetry}

The NPDGamma experiment [4] studies the reaction of polarized cold neutron capture on protons, and the prompt gamma emission from the deuteron product. A parity-violating spin dependence in the direction of gamma emission is predicted to exist on the order of $5 \cdot 10^{-8}$ by DDH. The differential cross-section for the emission of a prompt gamma ray goes as

$$
\frac{d \sigma}{d \Omega} \propto 1+A_{\gamma, P V} \cos \theta_{\gamma}+A_{\gamma, P C} \sin \theta_{\gamma}
$$

where $\theta_{\gamma}$ is the angle between the neutron spin and the direction of gamma emission, and the parityviolating asymmetry in terms of DDH parameters is given by [5]

$$
A_{\gamma, P V} \approx-0.107 f_{\pi}^{1}-0.001 h_{\rho}^{1}-0.004 h_{\omega}^{1},
$$

dominated by the pion coupling constant. The Parity-Conserving (PC) left/right asymmetry is represented by $A_{\gamma, P C}$.

The experiment is running at the Spallation Neutron Source (SNS) at ORNL on a cold neutron beamline. The beam is polarized to about $95 \%$ by a supermirror polarizer [6], and an RF spin rotator [7] alternates the polarization of the beam by rotating the neutron spin by 180 degrees. The proton target is a 16-liter cold $\mathrm{LH}_{2}$ vessel [8] with continuous flow through an ortho/para conversion catalyst to ensure a parahydrogen concentration of nearly $100 \%$, minimizing beam depolarization by orthohydrogen molecules [9]. To measure the asymmetry, an array of 48 CsI scintillators [10] surrounds the target (Figure 2).

The simplest calculation of the raw asymmetry as a function of detector is of the form

$$
A_{\text {raw }}=\frac{\sum_{i, \uparrow} N_{i}-\sum_{i, \downarrow} N_{i}}{\sum_{i, \uparrow} N_{i}+\sum_{i, \downarrow} N_{i}},
$$




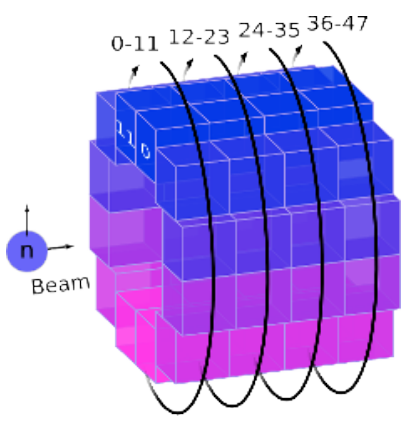

Figure 2. CsI scintillator array configuration - The liquid parahydrogen target is located inside the array along the neutron beam which is coaxial with the cylindrical array. The scintillation detectors are numbered from 0 to 47 increasing clockwise and along the beam direction. The symmetry of the array allows for calculation of the $n p \rightarrow d \gamma$ asymmetries with beam fluctuations removed.

where $i, \uparrow$ is the set of up-spin neutron pulses in a given spin sequence, and $N_{i}$ is the $i^{\text {th }}$ pulse timeaveraged detector signal. Though correct, this method is susceptible to fluctuations in beam power. More sophisticated methods use the detector array symmetry to remove beam fluctuations from statistical uncertainty.

In order to extract the PV and PC asymmetries in Eq. (1) and remove detector geometry dependence, geometrical factors are calculated for each detector. These factors take into account the configuration and acceptance of each scintillator, and the finite dimensions of the target. Two sets of factors are produced, one weighted by projection of the gamma detector's momentum acceptance onto the neutron polarization axis $\hat{\sigma_{n}}\left(G_{U D}^{d}\right)$, and the other $\left(G_{L R}^{d}\right)$ weighted by its projection onto $\hat{\sigma_{n}} \times \hat{k_{n}}$, where $\hat{k_{n}}$ is the direction of neutron momentum. The analysis is sensitive to asymmetries only in the plane of each circular ring, so that any asymmetry due to capture in hydrogen as a function of detector can be accounted for by a linear combination of the two orthogonal sets of geometrical factors. While accounting for background asymmetry $A_{b g}$, beam polarization $P_{n}$, and spin rotator efficiency $\epsilon_{s r}$, the geometrical factors are fit to the set of raw asymmetries as shown by the chi-square calculation below.

$$
\chi^{2}=\sum_{d} \frac{1}{\sigma_{d^{2}}^{2}}\left[\left(A_{\text {raw }}^{d}-A_{b g}^{d}\right)\left(P_{n} \epsilon_{s r}\right)^{-1}-\left(A_{\gamma, P V} G_{U D}^{d}+A_{\gamma, P C} G_{L R}^{d}\right)\right]^{2}
$$

\section{Current Status}

NPDGamma have been taking data with the liquid parahydrogen target since August 2012. During the commissioning phase, two key measurements were performed with larger nuclear targets. ${ }^{35} \mathrm{Cl}$ has a large $\left(10^{-5}\right)$ asymmetry which is well known, so a chlorine target was used to check the systematics and sensitivity of the detector. In addition, the largest background signal that will be encountered in the analysis of the hydrogen data is from aluminum. Measurements have been taken with an aluminum target and analysis is close to being completed.

In Figure 3, the measured raw asymmetry (see Eq. (3)) for the chlorine target is shown for a subset of the data. The plotted raw asymmetry includes both PV and PC contributions, though the chlorine asymmetry is dominated by large PV effects.

Figure 4 shows a subset of the recent data taken with the hydrogen target for approximately 11 days of beam with $850 \mathrm{~kW}$ power on the SNS spallation target. By using run time and experimental uncertainty estimates for new additional data, we expect to approach our goal uncertainty in the beginning of 2014. The experimental concept has been shown to be sound, and as the SNS facility and the NPDGamma experiment continue to run according to plan, we look forward to achieving a high precision measurement of the $n p \rightarrow d \gamma \mathrm{PV}$ asymmetry to place limitations on the weak interaction between nucleons carried by pions. 

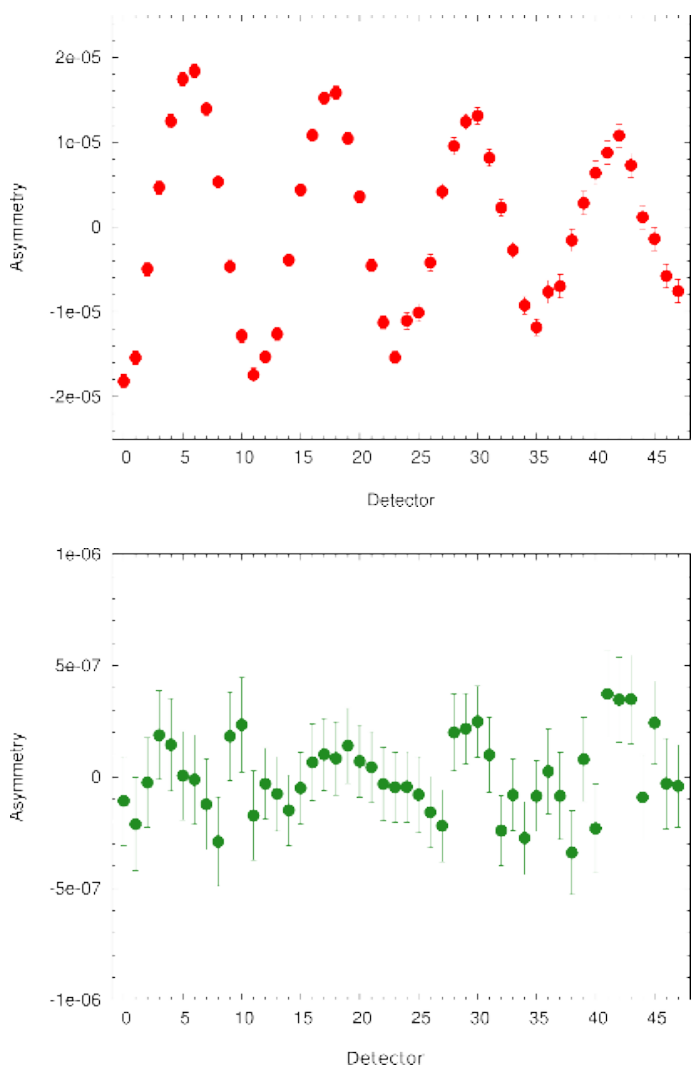

Figure 3. Preliminary raw ${ }^{35} \mathrm{Cl}$ asymmetry as a function of detector number (see Figure 2)
Figure 4. Early raw hydrogen asymmetry data as a function of detector number (see Figure 2) for 11 days of beam

\section{References}

[1] M.J. Ramsey-Musolf, S.A. Page, Ann. Rev. Nucl. Part. Sci. 56, 1 (2006)

[2] B. Desplanques, J.F. Donoghue, B.R. Holstein, Annals of Physics 124, 449 (1980)

[3] J. Wasem, Phys. Rev. C 85, 022501 (2012)

[4] M. Gericke, S. Page, D. Ramsay, R. Alarcon, S. Balascuta, L. Barron, J. Bowman, R. Carlini, W. Chen, T. Chupp et al., Nucl. Inst. Meth. A 611, 239 (2009)

[5] E.G. Adelberger, W.C. Haxton, Ann. Rev. Nucl. Part. Sci. 35, 501 (1985)

[6] S. Balascuta, R. Alarcon, S. Baeßler, G. Greene, A. Mietke, C. Crawford, R. Milburn, S. Penttila, J. Prince, J. Schädler, Nucl. Inst. Meth. A 671, 137 (2012)

[7] P.N. Seo, L. Barron-Palos, J. Bowman, T. Chupp, C. Crawford et al., Phys. Rev. ST Accel. Beams 11, 084701 (2008)

[8] S. Santra, L.B. Palos, C. Blessinger, J. Bowman, T. Chupp, S. Covrig, C. Crawford, M. Dabaghyan, J. Dadras, M. Dawkins et al., Nucl. Inst. Meth. A 620, 421 (2010)

[9] L. Barrón-Palos, R. Alarcon, S. Balascuta, C. Blessinger, J. Bowman, T. Chupp, S. Covrig, C. Crawford, M. Dabaghyan, J. Dadras et al., Nucl. Inst. Meth. A 659, 579 (2011)

[10] M. Gericke, C. Blessinger, J. Bowman, R. Gillis, J. Hartfield et al., Nucl. Inst. Meth. A 540, 328 (2005) 